\title{
Foreword
}

\section{Upon Assuming the Presidency of the Illuminating Engineering Institute of Japan}

\author{
Akira Sudo \\ President of IEIJ \\ Toshiba Corporation
}

By resolution at the Annual General Meeting of Members in May of this year, and by the board of directions at the time of that meeting, I have been elected president of the Illuminating Engineering Institute of Japan (IEIJ). Being named president of this historic academic society is a great honor, and at the same time I feel enormous responsibility.

\section{Paradigm shift of lighting field}

Although the field of lighting has a long history, in recent years there has been a transition from traditional light bulbs and fluorescent lamps to SSL (solid state lighting), such as LED lighting. Many members certainly sense that a dramatic paradigm shift is underway. Light source manufacturers, one after another, have discontinued production of general incandescent bulbs, and energy saving lighting luminaires that use LEDs are now exhibited at many appliance stores.

Furthermore, after the Great East Japan Earthquake LED lighting has played an important role in energy saving measures. Japan already has the world's highest adoption rate of LED lighting, the efficiency and service life of these LED light sources are still being improved day by day. Also, in terms of lighting techniques, various types of lighting that can be dimmed and color-mixed at will have been proposed. Such lighting is a radical change from the conventional so-called fixed and static lighting, and enables more aggressive proposals for lighting spaces.

Since such trends spread out widely, and due in part to the increasing number of common elements in light source units (lamps) and luminaires in LED lighting, the Japan Electric Lamp Manufacturers Association and the Japan Luminaires Associations have merged to form the Japan Lighting Manufacturers Association. In the business field, the barrier to entry has become low and the number of entrants continues to increase for this field and the display field.

How the IEIJ responds to these trends and changes, and whether it can continue to retain its attractiveness to many people, are major challenges for us. By all means, the IEIJ would like to continue to be a high-level source of information not only in hardware, in the physiological and psychological comfort of lighting, but also in the safety and security for public, in the field of crop-livestock farming and for other new industrial applications. We would like to activate research activities, education and information dissemination activities much more.

\section{Response to globalization}

This transition is progressing globally. The IEIJ would like to promote the hosting of international conferences and more active participation in them to encourage internationalization more strongly. The IEIJ is striving to disseminate the latest research and to provide training to young people through requesting greater participation from its members. Additionally, we would like to take the initiative to strengthen ties with other organizations in Japan and overseas, so that $R \& D$ results and academic achievements are reflected in international standardization activities, rather than being confined locally. 


\section{100-year anniversary and a new start}

In 2016, the IEIJ will celebrate its 100-year anniversary. In approaching this milestone, we are starting specific preparations this year for reviewing the history of lighting and studying the works of senior colleagues to reflect those to the present illuminating. With clarifying the present challenges of lighting technology and pursuing even better lighting through these activities, we would like to heighten the value of the presence of the IEIJ and therefore we request the cooperation of many individuals.

Above, I have described the paradigm shift, globalization and our 100-year anniversary. The IEIJ seems to be on the verge of entering a period of great change due to both internal and external factors. On April 1, 2012 the IEIJ moved to a general incorporated association and we newly established a subcommittee system.

I would like to make the IEIJ a place that fosters growth to enrich its members, and a place that provides great support and encouragement for academic activities.

I request further understanding and cooperation from all members in order to advance and promote the IEIJ. 